\title{
Left hippocampus sparing whole brain radiotherapy (WBRT): A planning study
}

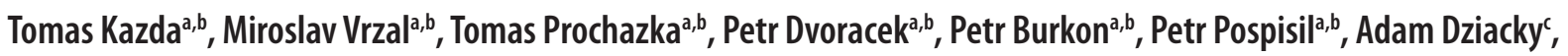 \\ Tomas Nikla, ${ }^{\mathrm{a},}$, Radim Jancalek ${ }^{\mathrm{d}, e}$, Pavel Slampa ${ }^{\mathrm{a}, \mathrm{b}, \mathrm{h}}$, Radek Lakomy ${ }^{\mathrm{f}, \mathrm{g}}$
}

\begin{abstract}
Aims. Unilateral sparing of the dominant (left) hippocampus during whole brain radiotherapy (WBRT) could mitigate cognitive decline, especially verbal memory, similar to the widely investigated bilateral hippocampus avoidance (HAWBRT). The aim of this planning study is dosimetrical comparison of HA-WBRT with only left hippocampus sparing (LHA-WBRT) plans.

Methods. HA-WBRT plans for 10 patients were prepared in accordance with RTOG 0933 trial and served as baseline for comparisons with several LHA-WBRT plans prepared with an effort: 1) to maintain the same left hippocampus dosimetry ("BEST PTV") and 2) to maintain same dosimetry in planning target volume as in HA-WBRT ("BEST LH").

Results. All HA-WBRT plans met RTOG 0933 protocol criteria with a mean Conformity index 1.09 and mean Homogeneity index (HI) 0.21. Mean right and left hippocampal D100\% was 7.8 Gy and $8.5 \mathrm{~Gy}$ and mean Dmax $14.0 \mathrm{~Gy}$ and $13.8 \mathrm{~Gy}$, respectively. "BEST PTV" plans reduced HI by $31.2 \%(P=0.005)$ which is mirrored by lower PTV_D2\% $(-0.8 \mathrm{~Gy}, P=0.005)$ and higher PTV_D98\% (+1.3 Gy, $P=0.005)$ as well as decreased optic pathway's Dmax by $1 \mathrm{~Gy}$. In "BEST LH", mean D100\% and Dmax for the left hippocampus were significantly reduced by $11.2 \%(P=0.005)$ and $10.9 \%(P=0.005)$ respectively. Conclusions. LHA-WBRT could improve target coverage and/or further decrease in dose to spared hippocampus. Future clinical trials must confirm whether statistically significant reduction in left hippocampal dose is also clinically significant.
\end{abstract}

Key words: brain metastases, hippocampus, memory, cognition, HA WBRT

Received: May 12, 2017; Accepted: June 20, 2017; Available online: July 20, 2017

https://doi.org/10.5507/bp.2017.031

\begin{abstract}
${ }^{a}$ Department of Radiation Oncology, Faculty of Medicine, Masaryk University, Kamenice 5, 62500 Brno, Czech Republic ${ }^{b}$ Department of Radiation Oncology, Masaryk Memorial Cancer Institute, Zluty kopec 7, 65653 Brno, Czech Republic 'Faculty of Medicine, Masaryk University, Kamenice 5, 62500 Brno, Czech Republic

${ }^{d}$ Department of Neurosurgery, St. Anne's University Hospital Brno and Faculty of Medicine, Masaryk University, Kamenice 5, 62500 Brno, Czech Republic

eDepartment of Neurosurgery, St. Anne's University Hospital Brno, Pekarska 53, 65691 Brno, Czech Republic

${ }^{f}$ Department of Comprehensive Cancer Care, Faculty of Medicine, Masaryk University, Kamenice 5, 625 00 Brno, Czech Republic ${ }^{9}$ Department of Comprehensive Cancer Care, Masaryk Memorial Cancer Institute, Zluty kopec 7, 65653 Brno, Czech Republic ${ }^{h}$ Regional Center for Applied Molecular Oncology (RECAMO), Masaryk Memorial Cancer Institute, Zluty kopec 7, 65653 Brno, Czech Republic Corresponding author: Radek Lakomy, e-mail: lakomy@mou.cz
\end{abstract}

\section{INTRODUCTION}

Hippocampal sparing during whole brain radiotherapy (WBRT) in an effort to preserve cognitive function is a currently widely investigated approach in palliative irradiation in patients with brain metastases unsuitable for local treatment ${ }^{1}$. Many planning studies have shown the dosimetric feasibility of hippocampus avoiding (HA) WBRT in different clinical situations using many different radiotherapy systems and techniques ${ }^{2-5}$. However, these studies must be considered as experimental until well designed clinical trials with appropriate endpoints definitely reveal the significant clinical benefit of these novel approaches ${ }^{2,6}$. Further, some reports suggest different post radiotherapy and/or post surgery changes in the two hippocampi. As a result, unilateral hippocampal sparing has been proposed ${ }^{7-11}$.

The most important neurocognitive domain affected by WBRT is memory. Radio-injury of the left, usually dominant, hippocampus may affect the formation of memory to a greater extent, in particular verbal memory ${ }^{9,11}$. Dosimetric consequences of potential unilateral (left) hippocampus sparing during WBRT are not known. We hypothesize that left hippocampal avoiding (LHA) during WBRT can possibly reduce the dose within the spared hippocampus as well as improve dose coverage of the remaining brain including the right hippocampus. Minimizing the dose to the least possible level is of high clinical relevance since higher hippocampal $\mathrm{D}_{100 \%}$ was shown to predict greater decline in some memory tests over time ${ }^{12,13}$. Also, the dose-response relationship between equivalent dose in 2-Gy fractions to $40 \%$ of the bilateral hippocampi $\left(\mathrm{EQD}_{2 \mathrm{~Gy}} 40 \%\right)$ and impairment in delayed recall at 18 months was described after fractionated stereotactic radiotherapy for benign or low-grade brain tumors ${ }^{12}$. The aim of this planning study was to compare HA-WBRT with LHA-WBRT treatment plans, paying particular attention to left hippocampal dosimetry and quantitatively describe the potential benefit of only one-sided hippocampal sparing. To the best our knowledge, this is the first report of the practical consequences of LHA-WBRT. 


\section{MATERIALS AND METHODS}

\section{Patients and contouring}

This retrospective planning study included ten consecutive patients with brain metastases and with available planning CT and MRI scans ( 9 women, mean age 54 years, 5/10 with lung cancer). Left and right hippocampi were separately contoured using the free available online RTOG contouring atlas as a reference ${ }^{14}$. A five millimeter margin around each hippocampus was created for the planning at risk volume (PRV) $\left(\right.$ ref. $\left.{ }^{15}\right)$. Other organs at risk (optic nerves, chiasm and lenses) were segmented according to recent contouring guidelines ${ }^{16}$. Planning target volume (PTV) contained the whole brain with $5 \mathrm{~mm}$ expansion to accommodate random setup uncertainty.

Three different treatment plans were created for each patient as described below (Fig. 1):

1) "standard" HA-WBRT serving as the baseline plan, 2) LHA-WBRT maintaining the same left hippocampal dosimetry as in the baseline plan (labeled as "BEST PTV") and 3) LHA-WBRT maintaining the same baseline plan's PTV's dosimetry (labeled as "BEST LH”). For "standard" HA-WBRT, both hippocampi_PRV were cropped out, while only the left hippocampus_PRV was cropped out for LHA-WBRT plans. Thus, these experimental plans included the entire right hippocampus within PTV.

\section{Experimental treatment plans}

All treatment plans were created utilizing Eclipse ${ }^{\mathrm{TM}}$ treatment planning system (Varian Medical Systems, Palo Alto, CA). The dose calculation was performed using the Anisotropic Analytical Algorithm (v11, Varian Medical Systems, Palo Alto, CA) with a $2.5 \mathrm{~mm}$ grid size and with the heterogeneity correction turned on. VMAT treatment plans were created for a TrueBeam STX with HD120 MLC (2.5 mm leaf width at isocenter). The $6 \mathrm{MV}$ photon beam model were used in all plans. The CT images which were used for planning were $3 \mathrm{~mm}$ slices.

Baseline plans for bilateral hippocampus sparing WBRT were created in accordance with online available RTOG 0933 (phase II trial assessing bilateral HA WBRT) study protocol criteria as follows: $90 \%$ of PTV receiving 10 x 3 Gy; PTV_D $2 \leq 37.5$ Gy, PTV_D ${ }_{98 \%} \geq 25$ Gy; D $\max$ of optic nerves or optic chiasm $\leq 37.5 \mathrm{~Gy}$, Hippocampal $\mathrm{D}_{100 \%} \leq 9$ Gy and Hippocampal $\left.\mathrm{D}_{\max } \leq 16 \mathrm{~Gy}\right)\left(\right.$ ref. $\left.^{13,15}\right)$. $\mathrm{D}_{\mathrm{x} \%}$ represents the dose received by $\mathrm{x} \%$ of specified structure. All treatment plans utilized three coplanar full arcs and one non-coplanar semi arc (couch angle 90 degrees and partial non-coplanar arc spanning 194 degrees (from 345 to 179 degrees)) in order to meet planning constraints criteria as much as possible.

\section{Dosimetric analysis}

The following parameters were reported for PTV: $\mathrm{V}_{100 \%}$, PTV_D $2 \%$, PTV_D ${ }_{98 \%}$, conformity index (CI) and homogeneity index (HI). CI was calculated based on the formula: $\left(\mathrm{V}_{95 \% \mathrm{Rx}} / \mathrm{V}_{\mathrm{PTV}}\right)$, where $\mathrm{V}_{95 \% \mathrm{Rx}}$ is the volume encompassed by $95 \%$ of the prescription isodose surface. This definition of CI is not strictly as in ICRU 62, since the

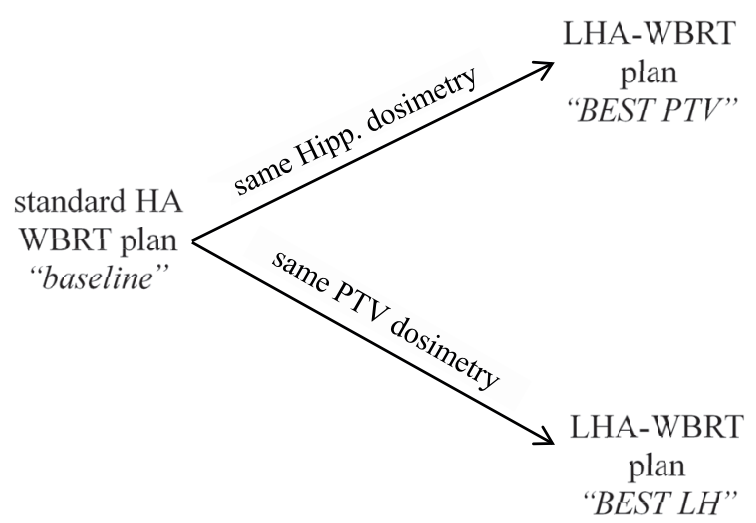

Fig. 1. Diagram of development of experimental treatment plans.

entire PTV is not always totally encompassed by the $95 \%$ isodose. Nevertheless, it remains a useful measure of the conformality of the proposed treatments ${ }^{17}$.

HI was calculated based on the formula: $\left(\left(\mathrm{D}_{2 \%}-\mathrm{D}_{98 \%}\right) /\right.$ $\mathrm{D}_{\text {median }}$ ) with zero indicating the ideal homogeneity since $\mathrm{D}_{2 \%}$ and $\mathrm{D}_{98 \%}$ are equal. $\mathrm{D}_{\max }$ was reported for optic structures. $\mathrm{D}_{40 \%}$ for the left hippocampus, converted to biologically equivalent doses in 2-Gy fractions (EQD2) assuming an $\alpha / \beta$ ratio of $2 \mathrm{~Gy}$, was recorded $\left(\mathrm{EQD}_{2}{ }^{2 \mathrm{~Gy}}\right.$ $40 \%$ ) (ref. ${ }^{12}$ ). All pertinent values were extracted from dose-volume histograms using an in house developed data mining software. Basic statistical methods including the Wilcoxon signed-rank test were used to obtain mean values and relative as well as absolute differences between HA-WBRT and experimental plans.

\section{RESULTS}

\section{Hippocampus avoiding-WBRT ("baseline") treatment plans}

HA-WBRT plans' characteristics are summarized in Table 1. All treatment plans met RTOG 0933 "per protocol criteria” for PTV coverage with mean CI 1.09 (range 1.08 - 1.1) and mean HI 0.21 (range 0.19 - 0.23). Mean value of near maximal dose in PTV was $\mathrm{D}_{2 \%}=33 \mathrm{~Gy}$ (RTOG 0933 limit is $\leq 37.5 \mathrm{~Gy}$ ) with mean near minimal dose in PTV $\mathrm{D}_{98 \%}=26.4$ Gy (RTOG 0933 limit is $\geq$ 25 Gy). All hippocampal dose-volume constraints were met ("per protocol criteria") with an exception in one patient where right as well as left hippocampal $\mathrm{D}_{100 \%}$ exceeded limit of $9 \mathrm{~Gy}$ (9.34 Gy and 10.07 Gy, respectively). The isodose treatment plan for one representative patient is presented in Fig. 2.

\section{Left HA-WBRT ("BEST LH") treatment plans}

For these experimental treatment plans, inverse planning was tailored to achieve the same PTV dosimetry as in "baseline" HA-WBRT. Resultant dosimetric characteristics are listed in table 2 along with absolute and relative differences between these data and mean parameters in "baseline" HA-WBRT. The right hippocampus is intentionally not spared leading to mean $\mathrm{D}_{100 \%}=30.1 \mathrm{~Gy}$. 
Table 1. Summary of treatment characteristics of HA-WBRT plans (effort to meet RTOG 0933 "per protocol” criteria).

\begin{tabular}{|c|c|c|}
\hline Target / organ at risk & $\begin{array}{l}\text { Mean (SD) } \\
\text { "baseline" }\end{array}$ & $\begin{array}{c}\text { RTOG } 0933 \\
\text { criteria }\end{array}$ \\
\hline \multicolumn{3}{|l|}{ PTV } \\
\hline $\mathrm{V}_{100 \%}$ & $90 \%(0)$ & $90 \%$ \\
\hline $\mathrm{D}_{2 \%}$ & 33 Gy $(0.08)$ & $\leq 37.5 \mathrm{~Gy}$ \\
\hline $\mathrm{D}_{98 \%}^{2 \%}$ & 26.4 Gy (0.29) & $\geq 25 \mathrm{~Gy}$ \\
\hline $\mathrm{CI}$ & $1.09(0.007)$ & \\
\hline HI & $0.21(0.01)$ & \\
\hline \multicolumn{3}{|l|}{ Optic nerves and chiasm } \\
\hline $\mathrm{D}_{\max }$ & 33.7 Gy $(0.48)$ & $\leq 37.5 \mathrm{~Gy}$ \\
\hline \multicolumn{3}{|l|}{ Hippocampus_right } \\
\hline $\mathrm{D}_{100 \%}$ & 7.8 Gy $(0.56)$ & $\leq 9 \mathrm{~Gy}$ \\
\hline $\mathrm{D}_{\max }$ & 14 Gy (0.37) & $\leq 16 \mathrm{~Gy}$ \\
\hline \multicolumn{3}{|l|}{ Hippocampus_left } \\
\hline $\mathrm{D}_{100 \%}$ & 8.5 Gy $(0.57)$ & $\leq 9 \mathrm{~Gy}$ \\
\hline $\mathrm{D}_{\max }$ & $13.8 \mathrm{~Gy}(0.21)$ & $\leq 16 \mathrm{~Gy}$ \\
\hline $\mathrm{EQD}_{2}{ }^{2 \mathrm{~Gy}} 40 \%$ & 8.24 Gy $(0.4)$ & \\
\hline
\end{tabular}

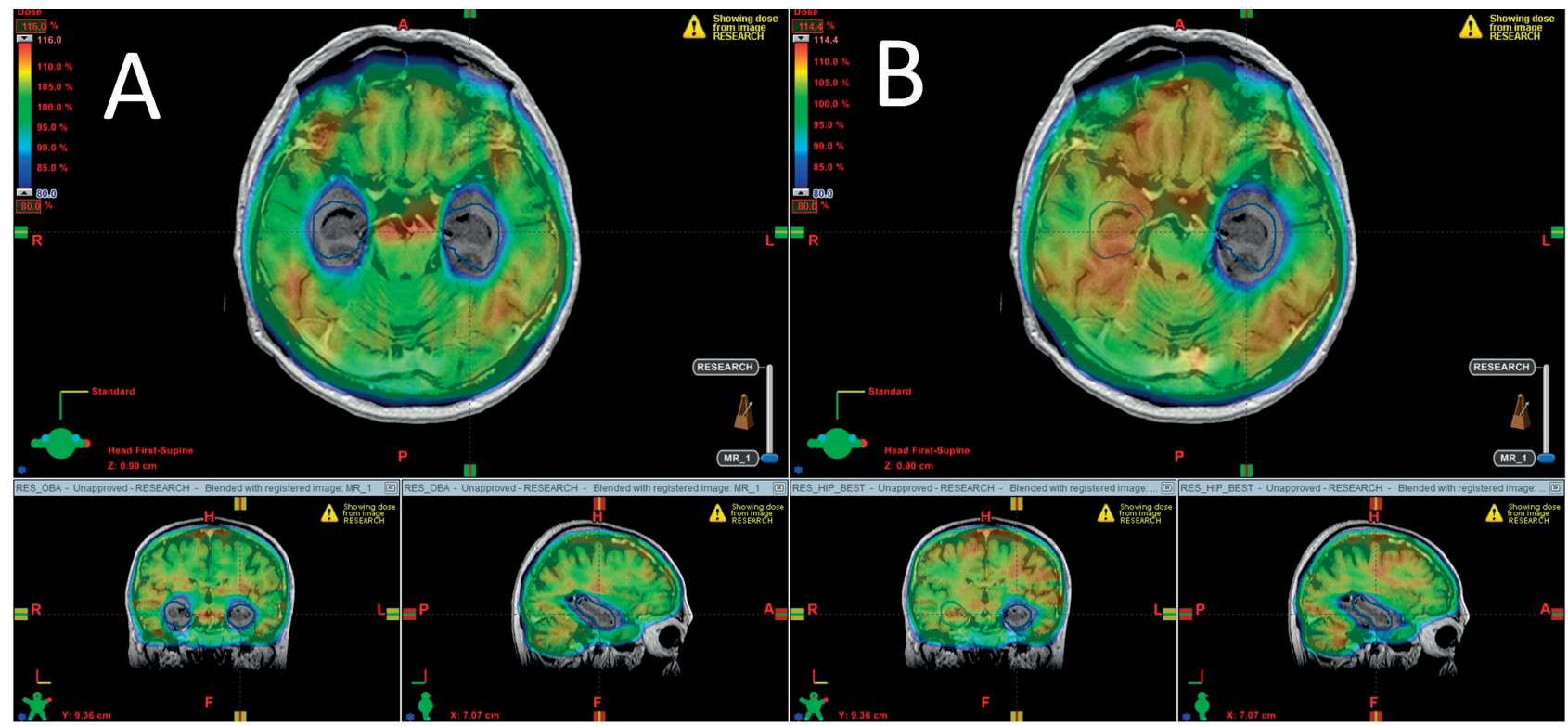

Fig. 2. Isodose treatment plan of representative HA-WBRT (A) and LHA WBRT ("BEST LH") for the same patient (B). Dose is displayed in color wash with minimal dose of $80 \%$ from prescribed dose.

Mean $\mathrm{D}_{100 \%}$ and $\mathrm{D}_{\max }$ for the left hippocampus were significantly reduced by $11.2 \%(P=0.005)$ and $10.9 \%(P=0.005)$ respectively. Moreover, left hippocampal EQD ${ }^{2 \mathrm{~Gy}} 40 \%$ was significantly reduced from a mean value $8.24 \mathrm{~Gy}$ in "baseline" to the mean value of 6.82 Gy in "BEST LH" experimental plan $(P=0.005)$. The isodose treatment plan for one representative patient is shown in Fig. 2.

\section{Left HA-WBRT ("BEST PTV") treatment plans}

For these experimental treatment plans, inverse planning was tailored to achieve the same left hippocampus dosimetry as in "baseline" HA-WBRT. Resultant dosimetric characteristics are listed in table 3 along with absolute and relative differences between these results and mean parameters in "baseline" HA-WBRT. Homogeneity of PTV irradiation was significantly improved with the same left hippocampal $\mathrm{D}_{100 \%}(P=0.92)$ and $\mathrm{D}_{\max }(P=0.39)$ as in "baseline" plans. HI was reduced by $31.2 \%(P=0.005)$ mirrored by lower PTV_D_ $(-0.8 \mathrm{~Gy}, P=0.005)$ and higher PTV_D ${ }_{98 \%}(+1.3 \mathrm{~Gy}, P=0.005)$. No difference was observed in conformity index.

\section{DISCUSSION}

The potential of further decrease of hippocampal irradiation during unilateral hippocampal sparing WBRT is described in this planning study. This concept makes it possible to significantly reduce unilateral hippocampal $\mathrm{D}_{100 \%}, \mathrm{D}_{\max }$ and $\mathrm{EQD}_{2}{ }^{2 \mathrm{~Gy}} 40 \%$ with comparable target coverage as in "standard" HA-WBRT. Vice versa, it is possible to significantly improve target coverage with ac- 
Table 2. Summary of treatment characteristics of Left HA-WBRT ("BEST LH”) treatment plans.

\begin{tabular}{|c|c|c|}
\hline Target / organ at risk & $\begin{array}{l}\text { Mean (SD) } \\
\text { "BEST LH" }\end{array}$ & $\begin{array}{l}\text { Absolute (relative) difference } \\
\text { to "baseline" HA-WBRT }\end{array}$ \\
\hline \multicolumn{3}{|l|}{ PTV } \\
\hline $\mathrm{V}_{100 \%}$ & $90 \%(0)$ & $0(0 \%)$ \\
\hline $\mathrm{D}_{2 \%}$ & 32.9 Gy (0.08) & -0.1 Gy $(-0.2 \%)$ \\
\hline $\mathrm{D}_{98 \%}$ & 27.1 Gy $(0.49)$ & +0.7 Gy $(+2.7 \%)$ \\
\hline CI & $1.1(0.004)$ & $+0.01(-0.7 \%)$ \\
\hline $\mathrm{HI}$ & $0.2(0.02)$ & $-0.03(-12.0 \%)$ \\
\hline \multicolumn{3}{|l|}{ Optic nerves and chiasm } \\
\hline $\mathrm{D}_{\max }$ & 33.3 Gy (0.37) & -0.5 Gy $(-1.4 \%)$ \\
\hline \multicolumn{3}{|l|}{ Hippocampus_right } \\
\hline $\mathrm{D}_{100 \%}$ & 30.1 Gy $(0.34)$ & $+22.3 \mathrm{~Gy}(+286.8 \%)$ \\
\hline $\mathrm{D}_{\max }$ & 32.9 Gy (0.39) & +19 Gy (+136\%) \\
\hline \multicolumn{3}{|l|}{ Hippocampus_left } \\
\hline $\mathrm{D}_{100 \%}$ & 7.6 Gy $(0.68)$ & -0.9 Gy $(-11.2 \%)$ \\
\hline $\mathrm{D}_{\max }$ & $12.3 \mathrm{~Gy}(0.19)$ & -1.5 Gy $(-10.9 \%)$ \\
\hline $\mathrm{EQD}_{2}^{2 \mathrm{~Gy}} 40 \%$ & 6.82 Gy $(0.54)$ & -1.43 Gy $(-17.4 \%)$ \\
\hline
\end{tabular}

Table 3. Summary of treatment characteristics of Left HA-WBRT ("BEST PTV”) treatment plans.

\begin{tabular}{|c|c|c|}
\hline Target / organ at risk & $\begin{array}{l}\text { Mean (SD) } \\
\text { "BEST PTV" }\end{array}$ & $\begin{array}{l}\text { Absolute (relative) difference } \\
\text { to "baseline" HA-WBRT }\end{array}$ \\
\hline \multicolumn{3}{|l|}{ PTV } \\
\hline $\mathrm{V}_{100 \%}$ & $90 \%(0)$ & $0(0 \%)$ \\
\hline $\mathrm{D}_{2 \%}$ & 32 Gy (0.07) & -0.8 Gy $(-2.4 \%)$ \\
\hline $\mathrm{D}_{98 \%}$ & $27.8 \mathrm{~Gy}(0.42)$ & $+1.3 \mathrm{~Gy}(+5 \%)$ \\
\hline CI & $1.09(0.008)$ & $+0.01(+0.55 \%)$ \\
\hline $\mathrm{HI}$ & $0.15(0.02)$ & $-0.07(-31.2 \%)$ \\
\hline \multicolumn{3}{|l|}{ Optic nerves and chiasm } \\
\hline $\mathrm{D}_{\max }$ & 32.7 Gy (0.39) & -1 Gy $(-3 \%)$ \\
\hline \multicolumn{3}{|l|}{ Hippocampus_right } \\
\hline $\mathrm{D}_{100 \%}$ & 29.6 Gy (0.29) & $+21.8 \mathrm{~Gy}(+280.7 \%)$ \\
\hline $\mathrm{D}_{\max }$ & $32.2 \mathrm{~Gy}(0.27)$ & +18.3 Gy (+131.1\%) \\
\hline \multicolumn{3}{|l|}{ Hippocampus_left } \\
\hline $\mathrm{D}_{100 \%}$ & 8.5 Gy $(0.54)$ & $+0.01 \mathrm{~Gy}(+0.2 \%)$ \\
\hline $\mathrm{D}_{\max }$ & 13.7 Gy $(0.33)$ & -0.1 Gy $(-0.8 \%)$ \\
\hline $\mathrm{EQD}_{2}^{2 \mathrm{~Gy}} 40 \%$ & 8.22 Gy (0.53) & -0.02 Gy $(-0.3 \%)$ \\
\hline
\end{tabular}

ceptance of the same unilateral hippocampal dosage as in "standard" HA-WBRT. Decreasing the hippocampal dose $\left(D_{100 \%}\right)$ to as low as possible was shown to predict less decline in some memory tests over time in patients irradiated for brain metastases ${ }^{12,13}$. More recently, white matter damage as well as thinning of the cerebral cortex after brain irradiation proved to be highly dose-dependent ${ }^{18,19}$. Hippocampus itself demonstrates radiation dose-dependent atrophy after treatment for brain tumors ${ }^{20,21}$. These dose-dependent brain changes are predicted to influence cognition and memory. Altogether, minimizing the dose within this critical part of the brain may be of high clinical relevance ${ }^{22}$. The risk of recurrence of brain metastases in undertreated regions is a reasonable trade-off especially in palliative irradiation.

Our previous study described significant decreases in hippocampal $\mathrm{N}$-acetylaspartate concentration (marker of neuronal density and viability) following WBRT (ref. ${ }^{10}$ ).
However, only changes in the left hippocampus positively correlated with the decline in memory function ${ }^{11}$. Another recent study of predictors for late neurocognitive dysfunction showed correlations of vascular dose-response in the left hippocampus in females with changes in memory function after radiotherapy ${ }^{9}$. Is it possible that only unilateral hippocampal radio-injury is responsible for subsequent neurocognitive decline? The functional anatomy of the cognitive domains suggests that verbal memory, the gold standard in evaluation of post radiotherapy cognitive impairment, often localize to the dominant hippocampus $^{7}$. Nevertheless, it would be useful to at first know, whether and to what extent this radiotherapy approach translates into improvement in radiotherapy treatment plans.

In order to demonstrate the potential of LHA-WBRT, we created two types of experimental plans with different optimalization intent. "BEST LH" treatment plans' 


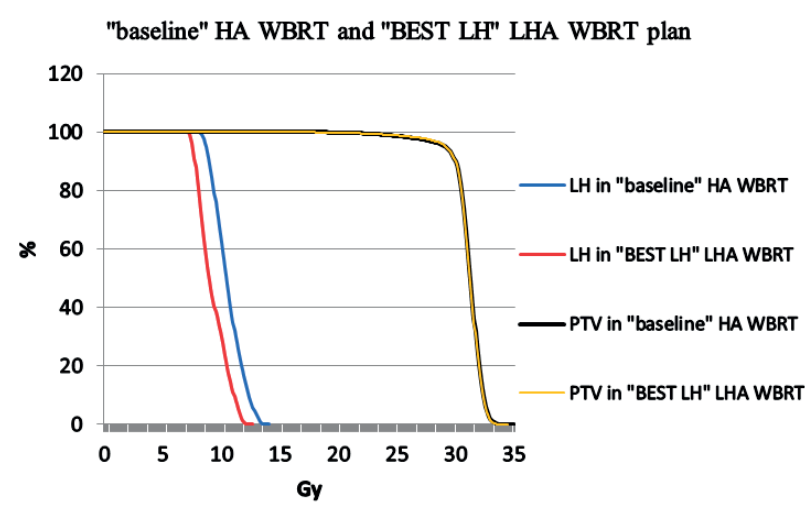

Fig. 3. Averaged dose-volume histogram from all patients presenting differences between "baseline" HA WBRT and "BEST LH” left hippocampus sparing WBRT treatment plans.

potential to further decrease left hippocampal dose with the same target coverage is showed in Fig. 3. The left hippocampal EQD ${ }^{2 \mathrm{~Gy}} 40 \%$ was significantly reduced from a mean value 8.24 Gy in "baseline" to mean $6.82 \mathrm{~Gy}$ in "BEST LH" experimental plan. This dosimetric parameter for both hippocampi was established by Gondi et al. with a threshold of $7.3 \mathrm{~Gy}$; irradiation surpassing this value is associated with long-term impairment in list-learning delayed verbal recall in patients with benign or low-grade adult gliomas ${ }^{12}$. "BEST LH" treatment optimization successfully undershot this threshold value. Although, we only assumed that the threshold value can be used for patients with brain metastases and for unilateral hippocampal sparing. Nevertheless, the slope of dose-response curve for $\mathrm{EQD}_{2}{ }^{2 \mathrm{~Gy}} 40 \%$ suggests that doses of around 6 to $8 \mathrm{~Gy}$ are highly clinically important ${ }^{12}$. For this reason, the decrease from $8.24 \mathrm{~Gy}$ to $6.82 \mathrm{~Gy}$ as observed in our planning study may be clinically relevant. Altogether, even if this reduction would not further decrease cognitive impairment (suggesting the dominant role of left hippocampus), with full irradiation of right hippocampus, the risk of subsequent development of hippocampal or perihippocampal metastases is reduced by one half. This risk is generally considered low because only $1.6 \%$ of brain metastasis extend to the hippocampus itself ${ }^{23}$.

There are several limitations in our study, which are mainly related to the nature of planning studies in general. Among others, comparison of PTV dosimetry between "baseline" HA-WBRT plan and two experimental LHAWBRT plans is biased due to different definition of PTV. This is because in LHA-WBRT plans, the right hippocampus was integrated to PTV. Another already mentioned limitation is in adaptation of hippocampal dose-volume constraints from studies of primary gliomas $\left(\mathrm{EQD}_{2}{ }^{2 \mathrm{~Gy}}\right.$ $40 \%)$.

The inherent limitation may be the fundamental question whether there is a role for WBRT in up-to-date treatment of brain metastases and with higher availability of systems for stereotactic radiosurgery ${ }^{24-26}$. From one perspective, the best technique for sparing the hippocampus is avoidance of WBRT itself and proceeding with stereo- tactic radiotherapy. Clearly, the role of WBRT is changing and more data from ongoing clinical trials are needed to establish the best treatment recommendations for defined subsets of patients ${ }^{27}$.

\section{CONCLUSION}

Unilateral hippocampal sparing during WBRT could improve target coverage and/or further reduce the dose to the spared hippocampus. Whether reduction in left hippocampus dosage as described in our planning study would further reduce post radiotherapy side effects warrants further research. Only future clinical trials can confirm whether statistically significant reduction in left hippocampal dose is also clinically significant.

\section{ABBREVIATIONS}

WBRT: whole brain radiotherapy; HA-WBRT: hippocampus avoiding whole brain radiotherapy; LHA-WBRT: left hippocampus avoiding whole brain radiotherapy; PTV: planning target volume; MRI magnetic resonance imaging; PRV planning organ at risk volume; VMAT: volumetric arc therapy.

Acknowledgement: This work was supported in part by the Ministry of Health, Czech Republic - Conceptual Development of Research Organization (MH CZ-DRO MMCI, 00209805) and project MEYS-NPS I-LO1413.

Author contributions: TK, MV, PP, RL: development of methodology and genereal research concept, plans evaluation, statistical analysis, manuscript writing; PD, TP: preparation of experimental treatment plan and cross approval; $\mathrm{PB}, \mathrm{AD}, \mathrm{TN}$ : contouring and evaluation of treatment plans, statistical analysis; RJ, PS: collaboration on manuscript writing, literature search and data interpretation. All authors contributed on the first draft's preparation and read and approved the final manuscript.

Conflict of interest statement: The authors state that there are no conflicts of interest regarding the publication of this article.

\section{REFERENCES}

1. Gondi V, Tomé WA, Mehta MP. Why avoid the hippocampus? A comprehensive review. Radiother Oncol 2010;97(3):370-6.

2. Kazda T, Jancalek R, Pospisil P, Sevela O, Prochazka T, Vrzal M, Burkon P, Slavik M, Hynkova L, Slampa P, Laack NN. Why and how to spare the hippocampus during brain radiotherapy: the developing role of hippocampal avoidance in cranial radiotherapy. Radiat Oncol 2014;9:139.

3. Awad R, Fogarty G, Hong A, Kelly P, Ng D, Santos D, Haydu L. Hippocampal avoidance with volumetric modulated arc therapy in melanoma brain metastases - the first Australian experience. Radiat Oncol 2013;8:62.

4. Marsh JC, Godbole R, Diaz AZ, Gielda BT, Turian JV. Sparing of the hippocampus, limbic circuit and neural stem cell compartment during partial brain radiotherapy for glioma: a dosimetric feasibility study. J Med Imaging Radiat Oncol 2011;55(4):442-9. 
5. Marsh JC, Ziel GE, Diaz AZ, Wendt JA, Gobole R, Turian JV. Integral dose delivered to normal brain with conventional intensity-modulated radiotherapy (IMRT) and helical tomotherapy IMRT during partial brain radiotherapy for high-grade gliomas with and without selective sparing of the hippocampus, limbic circuit and neural stem cell compartment. J Med Imaging Radiat Oncol 2013;57(3):378-83.

6. Kazda T, Pospíšil P, Doleželová H, Jančálek R, Slampa P. Whole brain radiotherapy: Consequences for personalized medicine. Rep Pract Oncol Radiother 2013;18(3):133-8.

7. Lee TM, Yip JT, Jones-Gotman M. Memory deficits after resection from left or right anterior temporal lobe in humans: A meta-analytic review. Epilepsia 2002;43(3):283-91.

8. Jalali R, Mallick I, Dutta D, Goswami S, Gupta T, Munshi A, Deshpande $D$, Sarin R. Factors influencing neurocognitive outcomes in young patients with benign and low-grade brain tumors treated with stereotactic conformal radiotherapy. Int J Radiat Oncol Biol Phys 2010;77(4):974-9.

9. Farjam R, Pramanik P, Aryal MP, Srinivasan A, Chapman $\mathrm{CH}$, Tsien Cl, Lawrence TS, Cao Y. A Radiation-Induced Hippocampal Vascular Injury Surrogate Marker Predicts Late Neurocognitive Dysfunction. Int J Radiat Oncol 2015;93(4):908-15.

10. Pospisil P, Kazda T, Bulik M, Dobiaskova M, Burkon P, Hynkova L, Slampa P, Jancalek R. Hippocampal proton MR spectroscopy as a novel approach in the assessment of radiation injury and the correlation to neurocognitive function impairment: initial experiences. Radiat Oncol 2015;10:211.

11. Pospisil P, Kazda T, Hynkova L, Bulik M, Dobiaskova M, Burkon P, Laack NN, Slampa P, Jancalek R. Post-WBRT cognitive impairment and hippocampal neuronal depletion measured by in vivo metabolic MR spectroscopy: Results of prospective investigational study. Radiother Oncol 2017;122(3):373-9.

12. Gondi V, Hermann BP, Mehta MP, Tomé WA. Hippocampal dosimetry predicts neurocognitive function impairment after fractionated stereotactic radiotherapy for benign or low-grade adult brain tumors. Int J Radiat Oncol Biol Phys 2013;85(2):348-54.

13. Gondi V, Pugh SL, Tomé WA, Caine C, Corn B, Kanner A, Rowley H, Kundapur V, DeNittis A, Greenspoon JN, Konski AA, Bauman GS Shah S, Shi W, Wendland M, Kachnic L, Mehta MP. Preservation of Memory With Conformal Avoidance of the Hippocampal Neural Stem-Cell Compartment During Whole-Brain Radiotherapy for Brain Metastases (RTOG 0933): A Phase II Multi-Institutional Trial. J Clin Oncol 2014;32(34):3810-6.

14. Hippocampal contouring: a contouring Atlas for RTOG 0933 Available from: [http://www.rtog.org/LinkClick.aspx?fileticket=59 vaU8vfgQc\%3d\&tabid=338]

15. Gondi V, Tolakanahalli R, Mehta MP, Tewatia D, Rowley H, Kuo JS, Khuntia D, Tomé WA. Hippocampal-sparing whole-brain radiotherapy: a "how-to" technique using helical tomotherapy and linear accelerator-based intensity-modulated radiotherapy. Int J Radiat Oncol Biol Phys 2010;78(4):1244-52.

16. Scoccianti S, Detti B, Gadda D, Greto D, Furfaro I, Meacci F
Simontacchi G, Di Brina L, Bonomo P, Giacomelli I, Meattini I, Mangoni M, Cappelli S, Cassani S, Talamonti C, Bordi L, Livi L. Organs at risk in the brain and their dose-constraints in adults and in children: A radiation oncologist's guide for delineation in everyday practice. Radiother Oncol 2015;114(2):230-8.

17. Bolsi A, Fogliata A, Cozzi L. Radiotherapy of small intracranial tumours with different advanced techniques using photon and proton beams: a treatment planning study. Radiother Oncol 2003;68(1):114.

18. Connor M, Karunamuni R, McDonald C, White N, Pettersson N Moiseenko V, Seibert T, Marshall D, Cervino L, Bartsch H, Kuperman J, Murzin V, Krishnan A, Farid N, Dale A, Hattangadi-Gluth J. Dosedependent white matter damage after brain radiotherapy. Radiother Oncol 2016;121(2):209-16.

19. Karunamuni $\mathrm{R}$, Bartsch $\mathrm{H}$, White NS, Moiseenko V, Carmona R, Marshall DC, Seibert TM, McDonald CR, Farid N, Krishnan A, Kuperman J, Mell L, Brewer JB, Dale AM, Hattangadi-Gluth JA.. DoseDependent Cortical Thinning After Partial Brain Irradiation in HighGrade Glioma. Int J Radiat Oncol Biol Phys 2016;94(2):297-304.

20. Seibert TM, Karunamuni R, Bartsch H, Kaifi S, Krishnan AP, Dalia Y, Burkeen J, Murzin V, Moiseenko V, Kuperman J, White NS, Brewer JB, Farid N, McDonald CR, Hattangadi-Gluth JA. Radiation Dose - Dependent Hippocampal Atrophy Detected With Longitudinal Volumetric Magnetic Resonance Imaging. Int J Radiat Oncol 2017;97(2):263-9.

21. Balentova S, Hajtmanova E, Filova B, Borbelyova V, Lehotsky J. Effect of Fractionated Irradiation on the Hippocampus in an Experimental Model. Klin Onkol 2015;28(3):191-9.

22. Tomé WA, Gökhan Ş, Gulinello ME, Brodin NP, Heard J, Mehler MF, Guha C. Hippocampal-dependent neurocognitive impairment following cranial irradiation observed in pre-clinical models: current knowledge and possible future directions. Br J Radiol. 2016;89(1057):20150762.

23. Lakomy R, Hynkova L, Pospisil P, Burkon P, Slavik M, Slampa $P$, Jancalek R, Kazda T. Patterns of failure after brain metastases radiotherapy: reflections on the importance for treatment and clinical trials reporting. Neoplasma 2017 Mar 3. [Epub ahead of print] doi: 10.4149/neo_2017_302

24. Fogarty GB, Hong A, Gondi V, Burmeister B, Jacobsen K, Lo S, Paton E, Shivalingam B, Thompson JF. Debate: adjuvant whole brain radiotherapy or not? More data is the wiser choice. BMC Cancer 2016; 16:372.

25. Mehta MP, Aoyama H, Gondi V. The Changing Role of Whole-Brain Radiotherapy: Demise or Time for Selective Usage? JAMA Oncol 2017 Jan 5 [Epub ahead of print] doi: 10.1001/jamaoncol.2016.5414

26. Matzenauer M, Vrana D, Vlachova Z, Cwiertka K, Kalita O, Melichar B. Radiotherapy management of brain metastases using conventional linear accelerator. Biomed Pap Med Fac Univ Palacky Olomouc Czech Repub 2016;160(3):412-6.

27. Soltys SG, Kirkpatrick JP, Laack NN, Kavanagh BD, Breneman JC, Shih HA. Is Less, More? The Evolving Role of Radiation Therapy for Brain Metastases. Int J Radiat Oncol 2015;92(5):963-6. 